\title{
INNOVATIONS IN CASTING TECHNIQUES FOR SINGLE CRYSTAL TURBINE BLADES OF SUPERALLOYS
}

\author{
Dexin $\mathrm{Ma}^{1,2}$, Fu Wang ${ }^{1}$, Qiang Wu ${ }^{3}$, Samuel Bogner ${ }^{1}$, Andreas Bührig-polaczek ${ }^{1}$ \\ ${ }^{1}$ Foundry Institute, RWTH Aachen University, Intzestr. 5, Aachen, 52072, Germany \\ ${ }^{2}$ State Key Laboratory of Long-Life High Temperature Materials, Deyang, 618000, China \\ ${ }^{3}$ ALD Vacuum Technologies GmbH, Wilhelm-Rohn-Str. 35, Hanau, 63450, Germany
}

Keywords: Superalloy, Bridgman process, Directional solidification, Single crystal, Turbine blade

\begin{abstract}
A series of novel casting techniques for single crystal (SC) blades of superalloys were presented which were developed in our institutions. Based on the Bridgman process, the Grain Continuator (GC) and the Heat Conductor (HC) techniques were developed to minimize the heat barrier which hinders the lateral SC growth from the blade body into the platform's extremities. To achieve symmetric thermal conditions for the SC solidification in the blade clusters, the Parallel Heating and Cooling (PHC) system was employed. The negative shadow effect in the current Bridgman process and the related defects were significantly reduced. The Dipping and Heaving (D\&H) technique using thin shell molds enables a high temperature gradient to be obtained for SC growth and freckle free solidification of the superalloy castings.
\end{abstract}

In terms of the analysis of the solidification processing in the complex turbine blades, a new concept of a three dimensional (3D), precise control of the SC growth was proposed. Corresponding to this, a Targeted Cooling and Heating (TCH) technique was developed to dynamically establish a proper thermal arrangement for the microscopic control of the SC growth in the critical areas of large industrial gas turbine (IGT) blades. Combining the TCH and D\&H Technology, a more effective and precise control of local SC growth can be achieved based on the significant improvement of macroscopic cooling conditions.

\section{Introduction}

SC turbine blades of superalloys are widely used in aero-engines and have been increasingly introduced into power generation turbines. The SC blades of superalloys are traditionally produced by using the well-known Bridgman process, in which a shell mold containing alloy melt is withdrawn from a heating zone through a baffle into a cooling zone. In this way a directional solidified (DS) $\mathrm{SC}$ structure will then be formed from the bottom to the top of the blades. However, due to the thick ceramic molds and the poor radiative cooling conditions, it is difficult to maintain a reasonably high temperature gradient which is necessary for the desired solidification structure.

Demand for a more efficient DS/SC casting having a higher temperature gradient led to the development of the modified Bridgman techniques: such as the well-known Liquid Metal Cooling (LMC) [1] and the Gas Cooling Casting (GCC) processes [2]. Although both casting techniques result in higher cooling rates and finer microstructures in comparison to the conventional Bridgman process, they are still not widely employed in the industrial field because of the high cost of equipment investment and complicated procedures as well as the minimal improvement in casting quality. Over the past few decades, unremitting efforts have been made to develop alternative processes for industrial production. Nevertheless, up to now there are few significant modifications to the SC casting being reported.

As blade designs become more complex and the size of the SC castings becomes larger, the formation of casting defects caused by geometrical features becomes an increasingly serious challenge. As the most serious casting defect, stray grains mainly occur at the platform's extremities because of the abrupt transition of the cross-section and the rapid cooling of the local melt. Figure 1 shows typical blade geometry and the schematic of the dendrite growth in the SC blade. In the blade body portion, the SC growth can be considered to be vertical. However, in the platform region, the SC growth becomes 3-dimensional (3D). During the solidification, the alloy melt in the lower extremities C, B and D successively becomes undercooled, as shown in Figure 1 (b). In

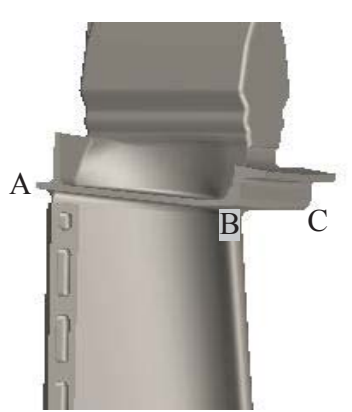

(a)

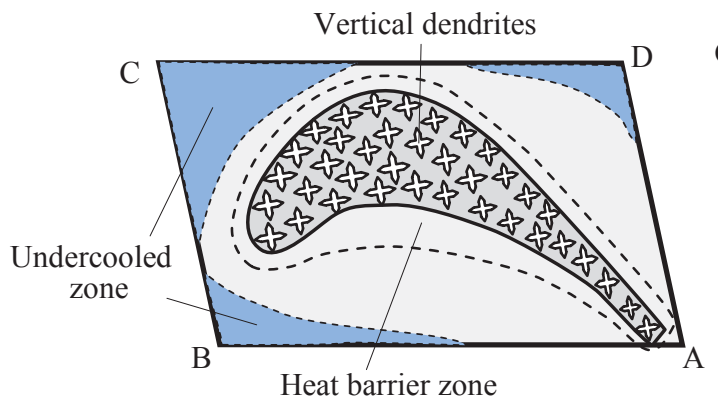

(b)

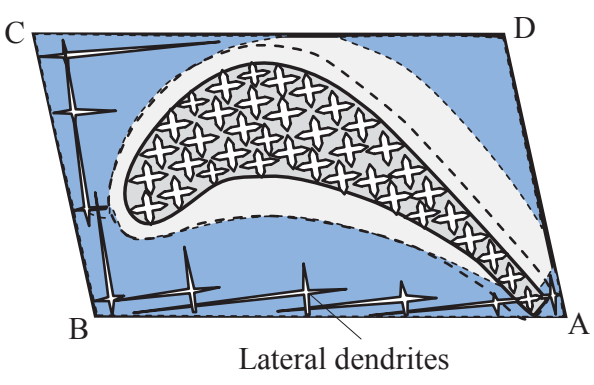

(c)

Figure 1. (a) typical blade geometry, (b) schematic of undercooling sequence and (c) SC solidification path in the transverse section of a blade platform. 
the geometric transition region between the blade body and the platform there is a nearly closed zone of heat barrier (Figure 1) caused by the poor cooling conditions. Here the alloy melt remains overheated longer and blocks the SC growth from the blade body into the undercooled platform. Only when the solidification front arrives at the highest extremity, A, at the trailing edge where no heat barrier exists, the primary dendrites of the SC can laterally grow from this position into the undercooled platform. The lateral SC growth in the platform progresses at first rapidly along the cold edges into the highly undercooled extremities (Figure 1 (c)), then turns inward towards the heat barrier zone with gradually reducing velocity, and then finally meets the dendrites coming directly from the blade body (Figure 2).

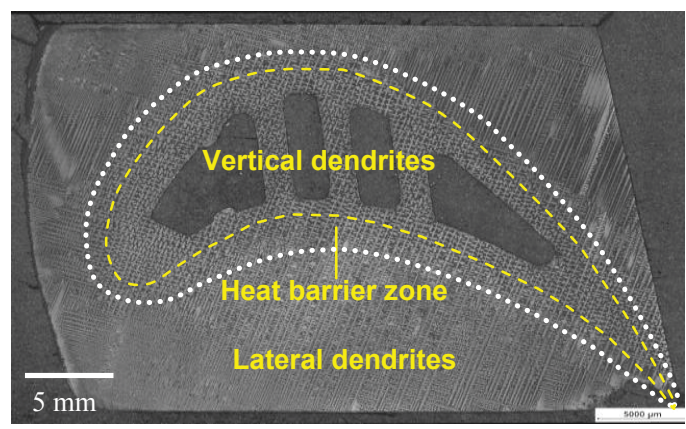

Figure 2. Transverse section of a CMSX-6 blade platform showing the 3-dimensional growth of the SC dendrites.

It should be noted, as shown in Figure 1, that the cooling sequence in the platform edge is $\mathrm{C} \rightarrow \mathrm{B} \rightarrow \mathrm{A}$. But the solidification sequence is in the opposite direction, namely $\mathrm{A} \rightarrow \mathrm{B} \rightarrow \mathrm{C}$. This thermal condition is inconsistent for stable and controllable SC growth. Even if the SC can tortuously grow into the entire platform, the quality of SC becomes inferior, due to the very inhomogeneous microstructure, as shown in Figure 2. Moreover, if the local undercooling at the extremities exceeds the undercoolability of the used alloy [3], stray grains will be formed before the arrival of the SC growth [4], as shown in Figure 3.

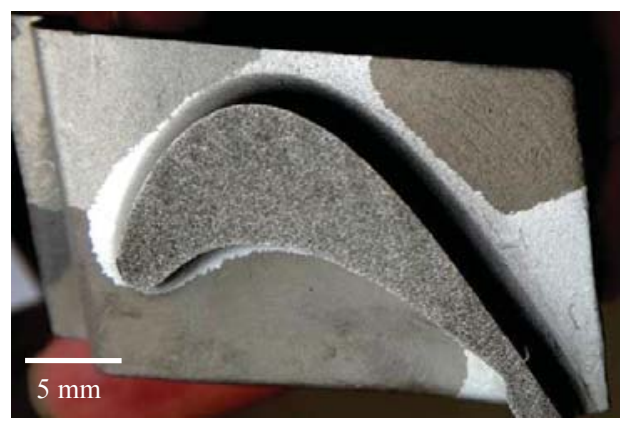

Figure 3. Turbine blade of a superalloy having low undercoolability, showing stray grains on the platform.

As stated above, the SC growth in a blade, especially in the platform region, is more complex than that in a solely vertical solidification process. Special efforts should be taken to properly guide and control the SC solidification along specific routes, rather than to generally intensify the cooling of the entire component. In fact, the cooling rate of the blade's extremities
(Figure 1-3) is already too high for the SC solidification in the conventional process, by further increasing the cooling rate will lead to more serious defect formation.

In the authors' institutions, the casting of superalloy components has long remained the focus of the investigation. In this paper, some approaches for improving the production technique of the $\mathrm{SC}$ blades will be presented in order to use the full potential of the $\mathrm{SC}$ components by optimizing the Bridgman process resulting in defect-free as-cast structure.

\section{Technique improvements}

\section{Grain Continuator (GC) Technique}

This technique was developed at the Foundry-Institute of RWTH Aachen University 20 years ago [5]. In order to circumvent the heat barrier zone, a bypass (continuator) can be established for transferring the SC to the platform's extremities (Figure 4 (a)). For this purpose, during the assembly of the wax patterns, a thin wax bar of a few millimeters in diameter can be additionally attached between the adapter above the grain selector and those platform extremities which are susceptible to form the stray grains. Owing to the better cooling conditions, the solidification front in this grain continuator progresses faster than that in the blade. The solidification front will reach the platform before the alloy melt in the extremities becomes undercooled. In this way the bypassed $\mathrm{SC}$ orientation can be transferred to the platform. However, during the crystal growth inside of the grain continuator, the orientation of the dendrite arms will twist differently to those in the blade section. As a result of this, the formation of subgrain boundaries is unavoidable (Figure 4 (b)). After casting, the added grain continuators have to be mechanically removed. Therefore, this technique cannot be employed for producing the casting parts which require a net shape and where no machining is permitted.

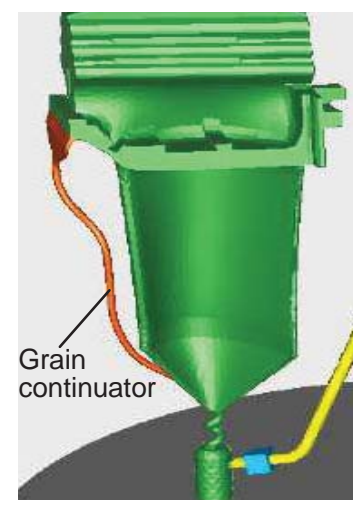

(a)

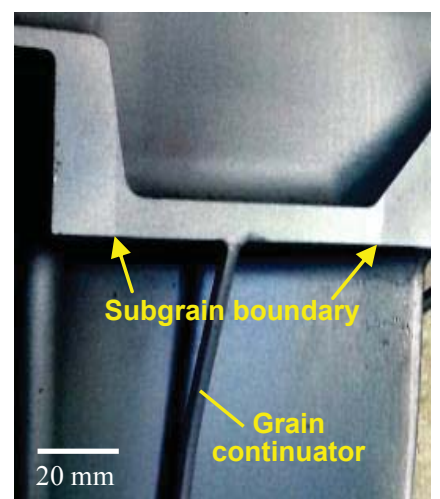

(b)
Figure 4. (a) the Grain Continuator (GC) technique employed to avoid stray grains and (b) the subgrain boundaries.

\section{Heat Conductor (HC) Technique}

Another method to suppress the formation of the stray grains at the platform extremities is the Heat Conductor (HC) technique [68]. It is believed, as shown in Figure 1 and Figure 5 (a), that a hot spot exists resulting from the poor local cooling condition at the inner corner of the cross-sectional transition regions. The heat barrier hinders the SC growth from the blade body portion into the platform. On the other hand, the outer extremity cools more 
rapidly because of the much thinner, local wall-thickness of the mold, where the stray grains prefer to form. In the HC technique, as shown in Figure 5 (a) and (b), a heat conductor is attached adjacent to the heat spot regions to minimize the heat barrier by improving the local heat extraction conditions. Graphite is used as $\mathrm{HC}$ material because of its extremely high thermal conductivity compared to ceramic mold. Moreover, graphite also has a high emission coefficient, which ensures effective heat radiation into the cold chamber of the Bridgman furnace. (a)

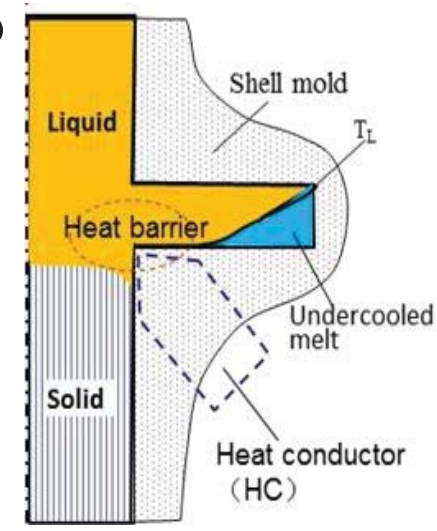

(b)

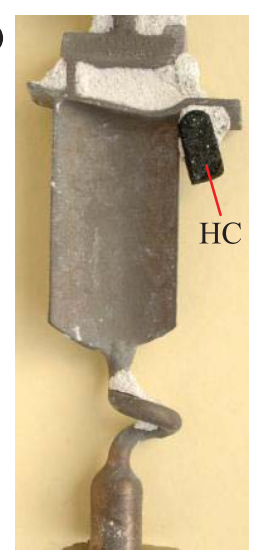

Figure 5. (a) schematic of the HC technique to reduce the heat barrier hindering the lateral SC growth, (b) A blade with attached $\mathrm{HC}$ after shake-out from the mold.
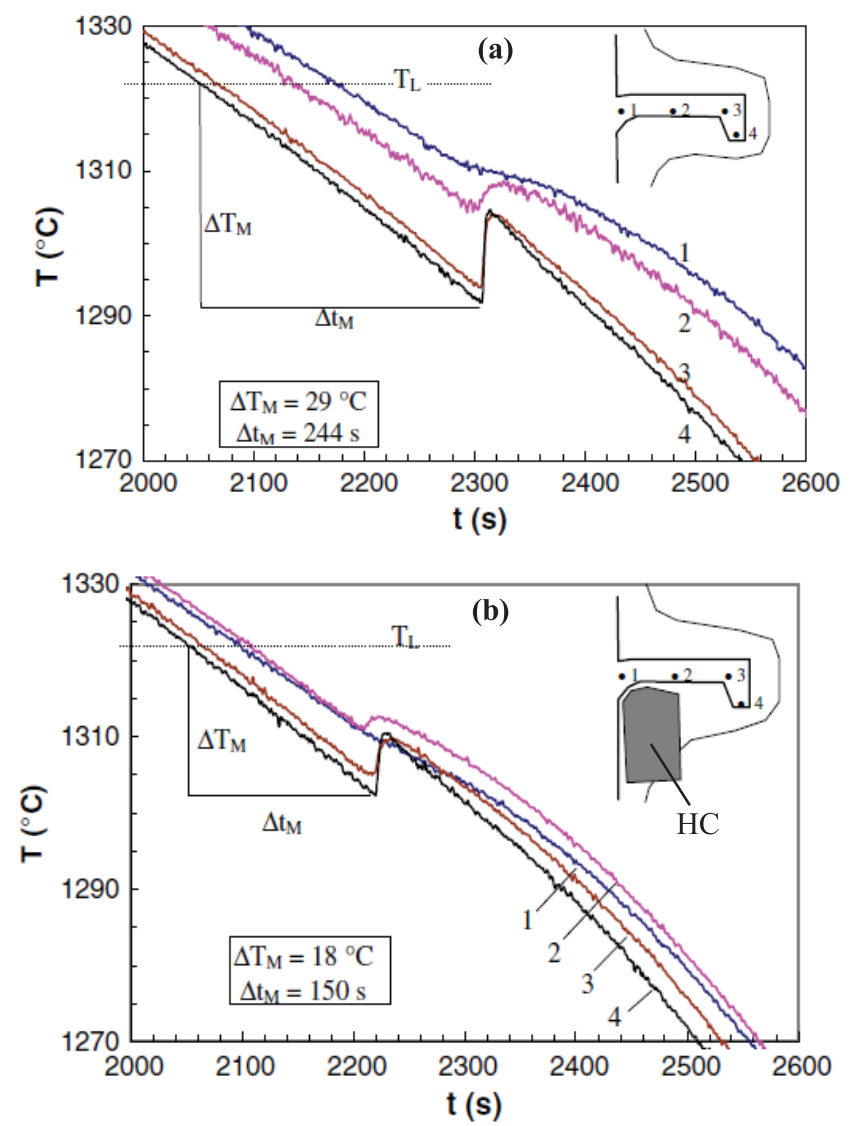

Figure 6. Cooling curves measured at the marked positions in the blade platforms: (a) conventional process, (b) with HC.
Figure 6 shows the cooling curves in the platform during solidification process, measured by the thermocouples at the marked positions. Without $\mathrm{HC}$, the maximum undercooling $\Delta \mathrm{T}_{\mathrm{M}}$ in the outer edge (position 4) is $29{ }^{\circ} \mathrm{C}$ (Figure 6 (a)), while with $\mathrm{HC}$ it is reduced to about $18{ }^{\circ} \mathrm{C}$ (Figure 6 (b)). The corresponding undercooling time $\Delta \mathrm{t}_{\mathrm{M}}$ is reduced from about 244 to 150 seconds by applying $\mathrm{HC}$ technique. Since the probability of grain nucleation in an alloy melt is dependent on the value of $\Delta \mathrm{T}_{\max }$ and $\Delta \mathrm{t}_{\max }$, the conclusion must be that the application of $\mathrm{HC}$ will significantly reduce the probability of stray grain formation.

In a series of casting experiments, the yield rate of acceptable blades was improved from $37 \%$ to $100 \%$ in comparison to the conventional process [8]. Figure 7 shows the typical dendritic structure of the platform extremities without and with HC. The results indicate that the $\mathrm{HC}$ technique can effectively reduce geometrically related stray grains. (a)

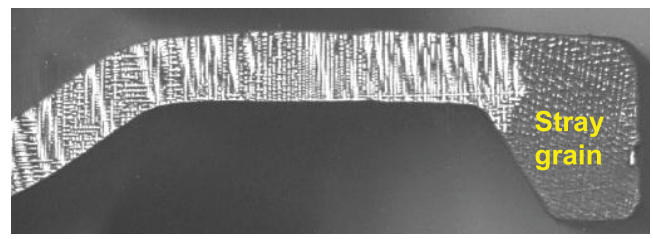

(b)

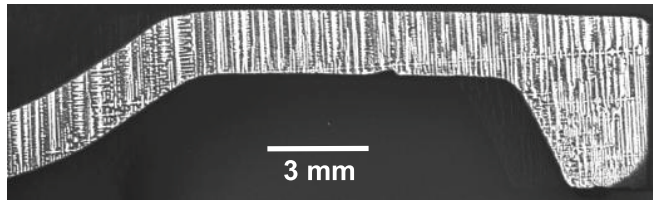

Figure 7. Typical grain structure of the platform extremities: (a) stray grain defect formed during conventional Bridgman process; (b) stray grain free $\mathrm{SC}$ structure achieved by applying $\mathrm{HC}$ technique.

In comparison to the Grain Continuator (GC) technique, a better monocrystallinity can be achieved by using the HC technique owing to the absence of the subgrain boundaries (Figure 4 (b)). In addition to this, this technique can be employed for any section irrespective of machining limitations since no additional materials has to be removed.

\section{$\underline{\text { Parallel Heating and Cooling (PHC) System }}$}

In a conventional Bridgman furnace, the heater and cooling chill have a cylindrical construction, as shown in Figure 8. Correspondingly, a shell mold cluster for a group of parts is arranged in a circle around a central rod. During the Bridgman process, the heating and cooling condition is asymmetrical in the cast parts due to different radiation conditions. Yu et al. [9] investigated the temperature distribution and temperature gradient in investment castings by using 3D model simulations based on the FEM method. The results showed an asymmetric temperature distribution and a curved liquid-solid interface. Napolitano et al. [10] pointed out that the formation of some types of grain defects, such as stray grains, was different on the two sides of the platform.

According to the cylindrical construction of the Bridgman-type furnace (Figure 8), the outside of the mold cluster can be immediately irradiated by the adjacent heater above the baffle. In the cooling zone below the baffle, this side can be effectively cooled by radiating heat to the adjacent chill ring. Therefore an 
obviously high thermal gradient for SC growth is provided on the outside of the mold cluster. In contrast, the heat transfer condition on the inward side of cluster facing the central rod is much poorer, inducing a shadow zone in the centre of the cluster (Figure 8).

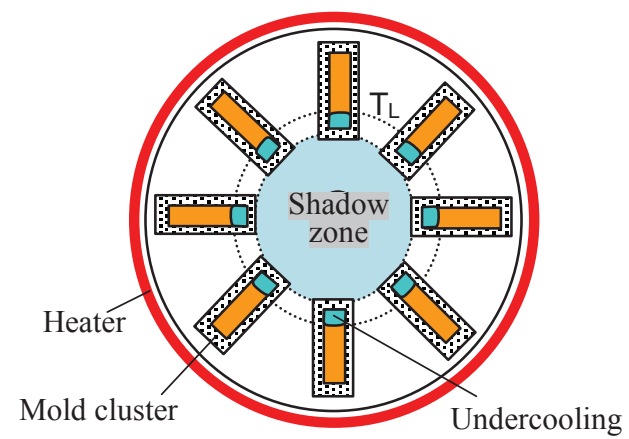

(a)

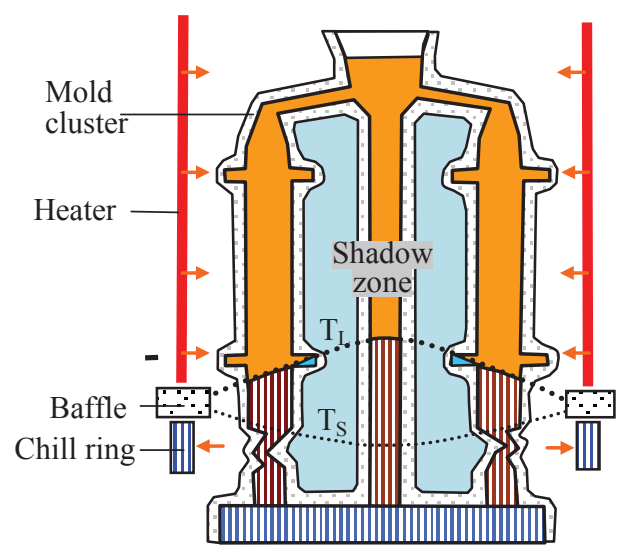

(b)

Figure 8. Sketch of the cylindrical Bridgman furnace currently used for manufacturing SC blades in clusters: (a) transverse section and (b) longitudinal section.

As the mold cluster is withdrawn downward, the cylindrical shadow zone passes through the heating and cooling zones without the thermal isolation of the baffle (Figure 8 (b)), similar to an opening at the bottom of furnace. This leads not only to the loss of energy but also, which is worse, to the decrease in thermal gradient and to an extension of the mushy zone. Therefore the solidification condition on the inward side of the cluster becomes very poor for SC growth. The metallographic examination indicated a high susceptibility to stray grains $[6,7,11]$ and freckles [12-14] in the shadow zone, while the heater side revealed a significantly lower tendency for these defects.

As stated above, the negative shadow effect become a typical disadvantage of the conventional Bridgman process. Particularly in the large furnaces, the cross section area of the cylindrical shadow zone correspondingly increases with that of heating chamber. Due to the wide opening of the furnace base, little isolation exists between the heating and cooling zone, leading to a more pronounced shadow effect. Therefore the conventional Bridgman furnace cannot be built too large because the productivity cannot be simply increased as expected. Instead, the furnace size should be properly decreased in order to improve the quality of the SC blades.
To remove the negative shadow effect and achieve symmetric thermal conditions for solidifying the SC blades, the Parallel Heating and Cooling (PHC) system was developed [15]. Figure 9 schematically shows the corresponding furnace construction having a double-row-arrangement. In fact, more rows of heaters and cooling chills can be constructed in parallel to increase the casting productivity without any loss of SC quality due to the absence of the shadow zone appearing in the conventional Bridgman process.

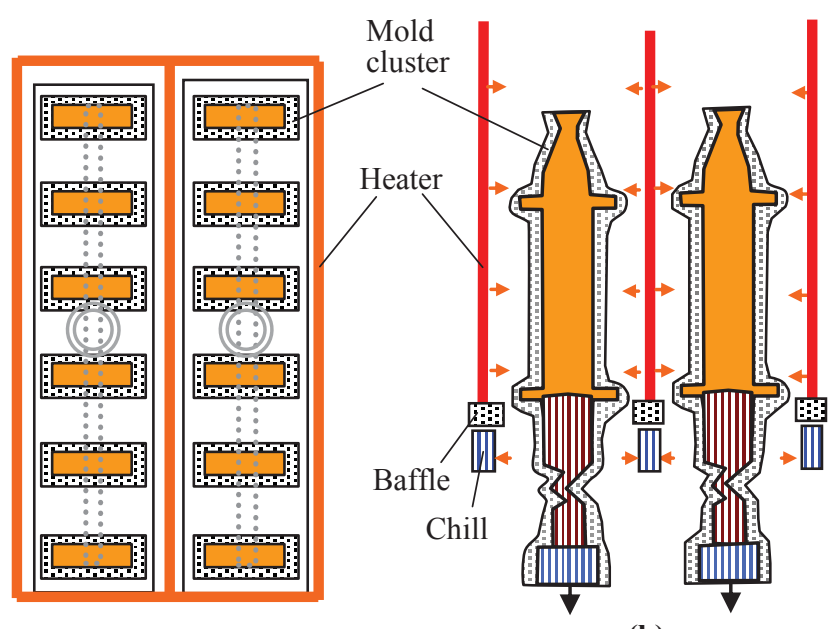

(a)

(b)

Figure 9. Sketch of the Parallel Heating and Cooling (PHC) system. (a) transverse section and (b) longitudinal section.

In comparison to the conventional process, the new furnace features the linear heater and cooling component. Correspondingly, the casting parts are aligned on a rectangular chill plate and can be symmetrically heated by the linear heater from both sides. After casting, the parts will be drawn downwards into the cooling area and symmetrically cooled. The symmetric heating and cooling provides uniform thermal conditions with the benefit for optimizing the microstructure and avoiding solidification defects. Due to the disappearance of the cylindrical shadow zone (Figure 8), the heating and cooling zones are much better isolated, leading to an increase in thermal gradient for SC solidification.

Figure 10 (a) exhibits the simulated temperature development at the platform of a dummy blade during the conventional Bridgman process. As a result of the asymmetric heating and cooling, the temperature distribution shows non-uniformity in the geometrically symmetric specimen. On the inward side facing the central rod, the melt is cooled down to the liquidus temperature earlier. Consequently, before the liquidus isotherm arrives at the platform, the melt on inner side is already undercooled. The maximum undercooling is calculated to be $15 \mathrm{~K}$. New grains may nucleate if the local undercooling sufficiently exceeds the critical undercooling for nucleation: namely the undercoolability of the used alloy [3].

In the specimen solidified in the PHC System, Figure 10 (b), the temperature distribution is symmetric due to the same heating and cooling condition on both sides. According to the simulation, the maximum undercooling is reduced to $8 \mathrm{~K}$, much lower than that 
in Figure 10 (a). The tendency for stray grain formation is then significantly reduced.

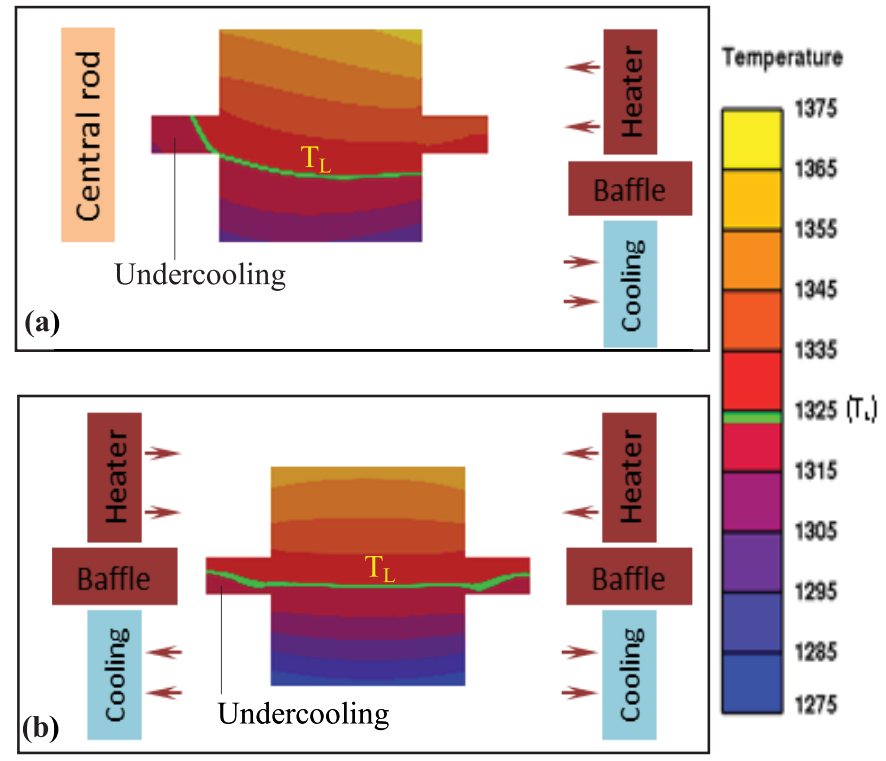

Figure 10. Simulated temperature distribution in a platform for (a) the conventional Bridgman and (b) the PHC process. The $\mathrm{T}_{\mathrm{L}^{-}}$ curve shows the liquidus isothermal.

(a)

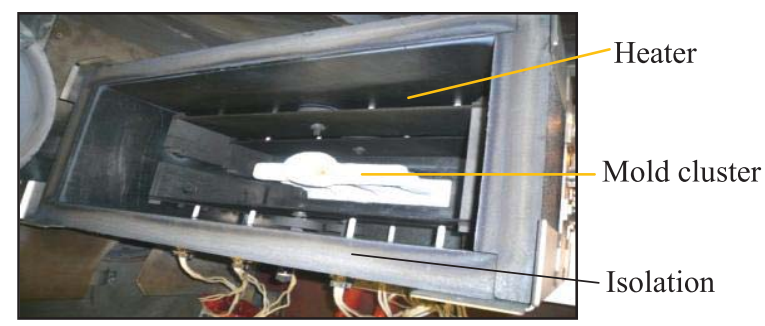

(b)

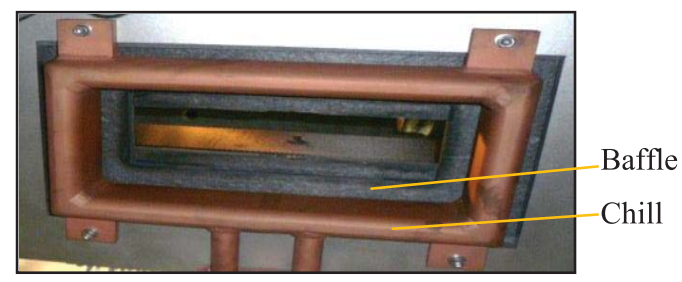

(c)

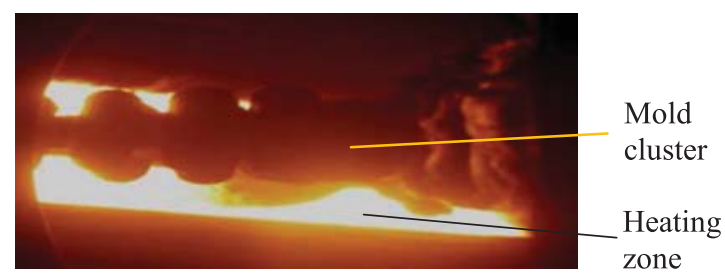

Figure 11. Pilot furnace with Parallel Heating and Cooling (PHC) System: (a) top view, (b) bottom view and (c) bottom view during casting experiment, showing the mold cluster being withdrawn from the heating zone.

Figure 11 shows a pilot furnace having a single row arrangement for the preliminary experiments. The casting cluster is linearly arranged so that both sides of a shell mold can be symmetrically heated in the heating zone and cooled in the cooling zone. For the casting experiments the superalloy IN939 was selected on account of its stronger tendency to form stray grains [3, 7]. After pouring the alloy melt into the preheated shell mold mounted on the chill plate, it was then withdrawn from the hot zone into the cold zone through the baffle at a velocity of $3.0 \mathrm{~mm} / \mathrm{min}$. For comparison, the corresponding experiments were also carried out in the conventional furnace.

A simplified turbine blade geometry was used for the experiments (Figure 12). It consists of a plate as blade body and a number of protrusions as platforms, extending $10 \mathrm{~mm}$ on each side from the blade body. Usually, there are one or two platforms in the turbine blades. However, three steps of platforms are designed here to investigate stray grain formation more effectively since the stray grains occur particularly at platforms.

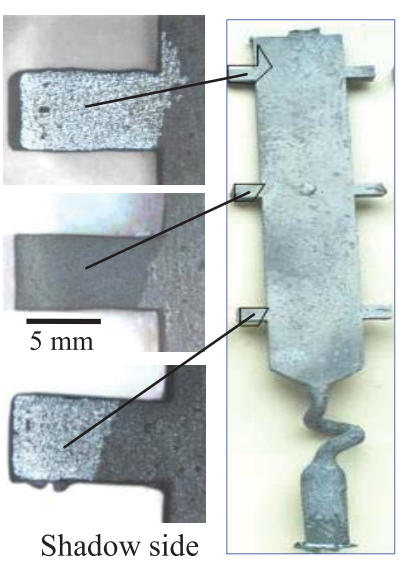

(a)

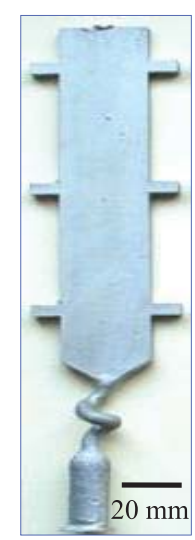

(b)
Figure 12. Typical macrostructure of dummy blades solidified (a) in a conventional Bridgman process revealing stray grain formation on the shadow (left) side of the platforms, while (b) the $\mathrm{PHC}$ process provides a nearly defect free as-cast structure.

The metallographic examination exhibited a significant difference in the stray grain formation. Figure 12 (a) shows the typical macrostructure of a specimen formed in the conventional Bridgman process. Stray grains are observed mainly on the shadow side of the platforms facing the central rod. On the outer side facing the heater, the platforms show a significantly lower tendency to stray grain formation. A comparably good result is also achieved for both sides of the blades cast in the PHC process (Figure 12 (b)).

The volume quota $Q_{S}$ is employed to evaluate the stray grain ratio. A quota value of $1(100 \%)$ means full occupation by stray grains, while a quota value of 0 indicates a platform completely free of stray grains. In the conventional process, the quota of the casting defect stay grain is $49 \%$, in which the $\mathrm{Q}_{\mathrm{S}}$ on the inner side is 15 times higher than that on the outer side, indicating a much higher risk of stray grain formation.

As expect, the stray grain formation is suppressed effectively by minimizing or even eliminating the asymmetric thermal condition. By using the PHC System, there are few stray grains found in the specimens, Figure 12 (b). Due to improving the symmetry of the thermal condition, both sides of the specimens show a minimal tendency for stray grain formation. The stray grain ratio in the specimens is measured to be significantly reduced from $49 \%$ to 
$6 \%$, in comparison to the conventional Bridgman process. Therefore, the feasibility for improving the quality of the SC components by using the PHC System is clearly confirmed.

\section{Dipping and Heaving (D\&H) Technology}

Using the conventional Bridgman processes it is difficult to maintain a reasonably high temperature gradient for SC solidification. This is mainly due to the following reasons: (1) ineffective radiative heat exchange, (2) unclosed baffle isolation between heating and cooling zones, (3) large thermal resistance in thick ceramic molds. To mitigate these limitations the DippingHeaving (D\&H) technology, previously also called Thin Shell Casting (TSC) [16] or Downward Directional Solidification
Process (DWDS) [17], was developed. In the D\&H process, as shown in Figure 13, superalloys are overheated to a stable temperature in a crucible and covered with hollow ceramic beads which function as a dynamic baffle. Ceramic molds, having SC seeds or a selector connected to a chill at one end, are dipped into the alloy's melt through the dynamic baffle. To prevent the entrance of the baffle's beads into the mold, its other end is sealed by a stopper made from the same alloy. When the stopper has melted, the alloy melt flows into the mold and comes into contact with the seed or the chill-plate. After a portion of the seed has melted and a steady-state thermal condition is reached, the mold is pulled up from the melt bath at a specified withdrawal rate and cooled by gas (argon). In this way, a SC component is formed during the downward solidification process.

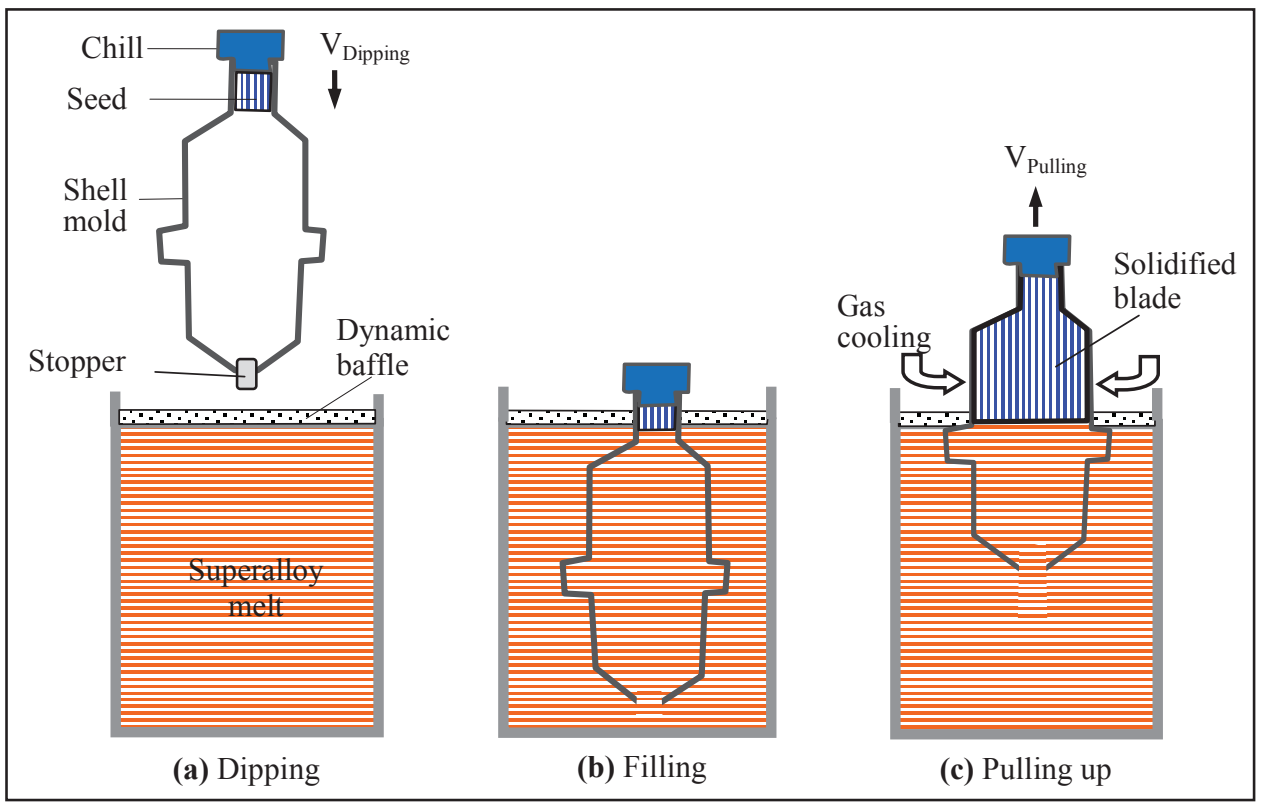

Figure 13. Illustration of the D\&H process: (a) dipping the shell mold into the melt bath, (b) mold filling, (c) pulling up the mold to perform the downward solidification.

In the D\&H process, owing to a balance of the hydrostatic pressure exerted by the melt between the inside and outside of the mold, the mold's wall-thickness can be reduced to only approximately $1 \mathrm{~mm}$, which is merely used to retain the casting's shape, as shown in Figure 14.

In a previous investigation, the SC dummy blades of Al-alloys were cast and directionally solidified under non-vacuum condition using D\&H technology, as shown in Figure 15 (a). A double-neck selector was employed in the process instead of a three dimensional (3D) helix in order to increase the mold filling capacity and the heat flux through the selector. The application of gas cooling enables an efficient heat extraction from the solidified component to the surrounding. After the solidification, the blades were shaken out. Figure 15 (b) shows an as-cast blade having residual eggshell-like ceramic shell mold on its surface. After sand blasting, the blade was macro-etched to detect the grain structure on the blade surface (Figure 15 (c)). In the starter section at the top, the grain boundaries between the columnar grains can be clearly recognized. Behind the grain selection, a SC structure was achieved throughout the entire blade. A number of SC blades made of Al-alloys were produced by repeating the procedure

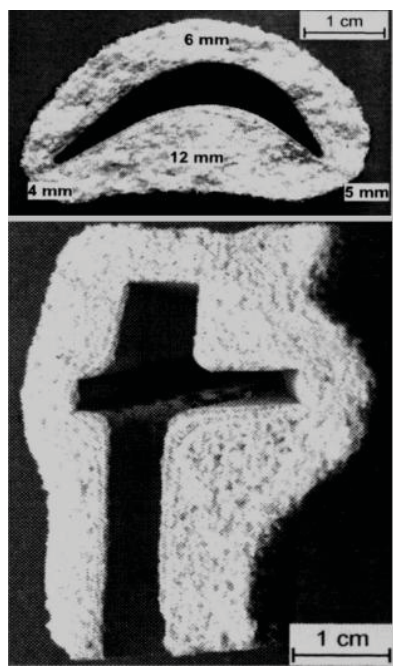

(a)

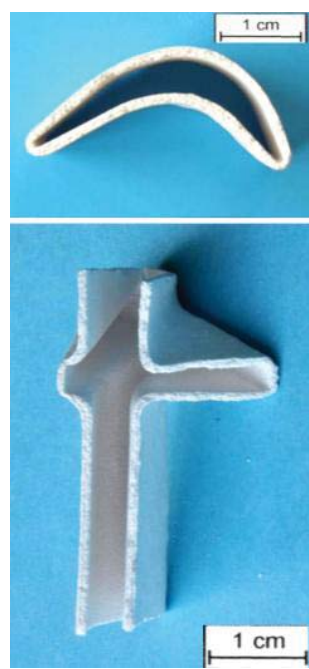

(b)
Figure 14. Wall-thickness of shell molds for production of turbine blades in (a) Bridgman and (b) D\&H process. 


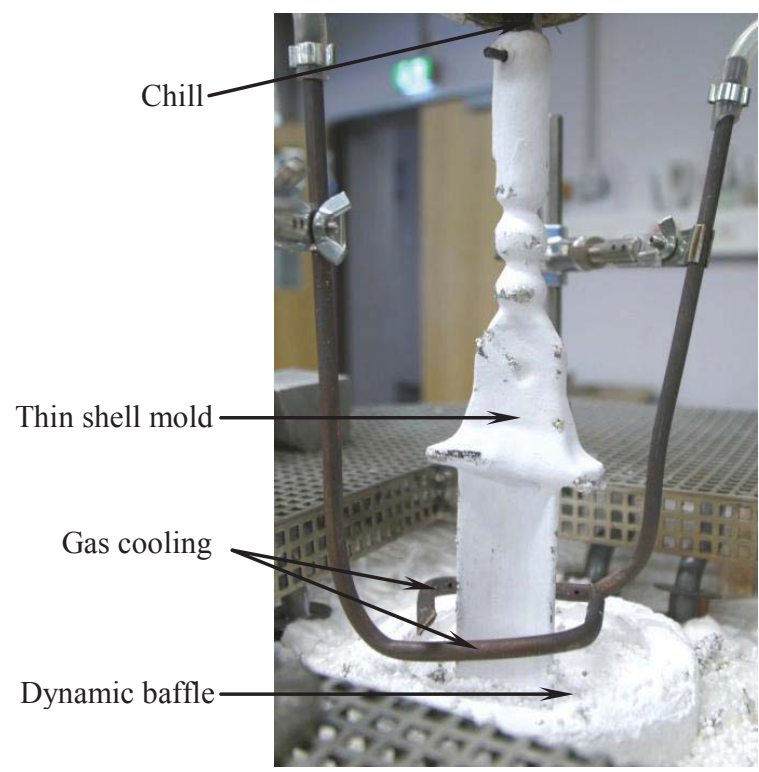

(a)

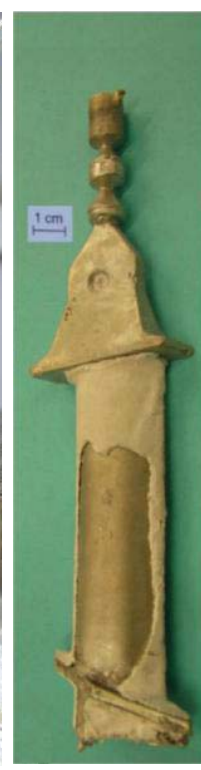

(b)

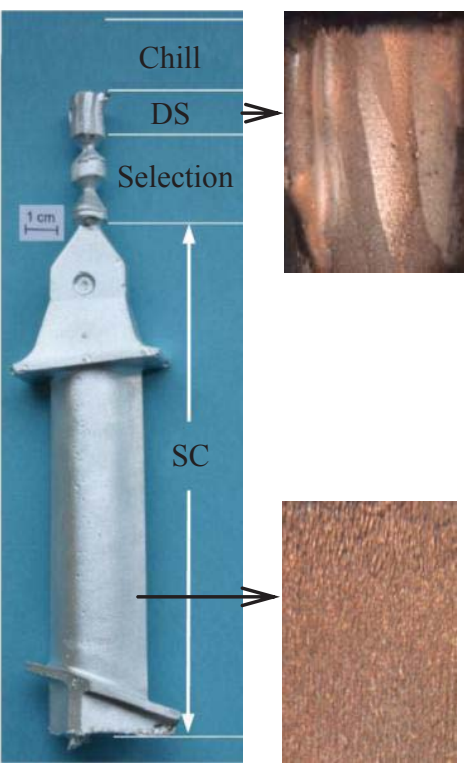

(c)

Figure 15. (a) D\&H (dipping and heaving) experiment using a thin shell to manufacture a SC blade of Al-alloys under non-vacuum conditions, (b) as-cast blade with residual shell and (c) macro- and microstructure of the SC blade.

of the D\&H technology in order to confirm the feasibility of this new process.

Recently, the D\&H technology was employed to produce SC blades of superalloys under protective argon gas [18]. Figure 16 (a) shows a SC blade of superalloy CMSX-4 produced using D\&H and a seeding technique. During the solidification the thermal gradient $(G)$ was measured to be about seven times higher than that in the Bridgman process. As a result, a refined microstructure was obtained (Figure 16 (b)). The structure parameters, such as the primary dendrite spacing $\lambda_{1}$, the average size of the $\gamma / \gamma^{\prime}$ eutectic pools and the $\gamma^{\prime}$ precipitates, and the volume fraction of the micropores, were significantly reduced, as listed in Table I.

Table I. Comparison of characteristic parameters between the Bridgman and D\&H processes

\begin{tabular}{|l|c|c|c|c|c|}
\hline & $\begin{array}{c}\mathrm{G} \\
(\mathrm{K} / \mathrm{mm})\end{array}$ & $\begin{array}{c}\lambda_{1} \\
(\mu \mathrm{m})\end{array}$ & $\begin{array}{c}\gamma / \gamma^{\prime} \\
\left(\mu \mathrm{m}^{2}\right)\end{array}$ & $\begin{array}{c}\gamma^{\prime} \\
(\mu \mathrm{m})\end{array}$ & $\begin{array}{c}\text { Porosity } \\
(\text { vol. \%) }\end{array}$ \\
\hline Bridgman & 2.2 & 445.6 & 1544.2 & 0.65 & 0.13 \\
\hline D\&H & 14.2 & 299.3 & 346.9 & 0.30 & 0.02 \\
\hline
\end{tabular}

In the future, a large vacuum furnace for $\mathrm{D} \& \mathrm{H}$ process is to be built, which is capable of both producing large IGT blades and also aero-turbine blades in clusters for high productivity. Furthermore, a molten slag will be used as a floating baffle material, so that the melt bath can be not only better isolated but also cleaned during the process, similar to the ElectroslagRemelting (ESR) process.

The D\&H technique has numerous advantages: Due to the use of extremely thin shell molds and the application of gas cooling, an efficient heat extraction from the solidifying component can be achieved in comparison to the conventional and other modified Bridgman techniques. The heating and cooling zones can be well isolated by using the dynamic baffle resulting in a higher thermal gradient at the solidification front.
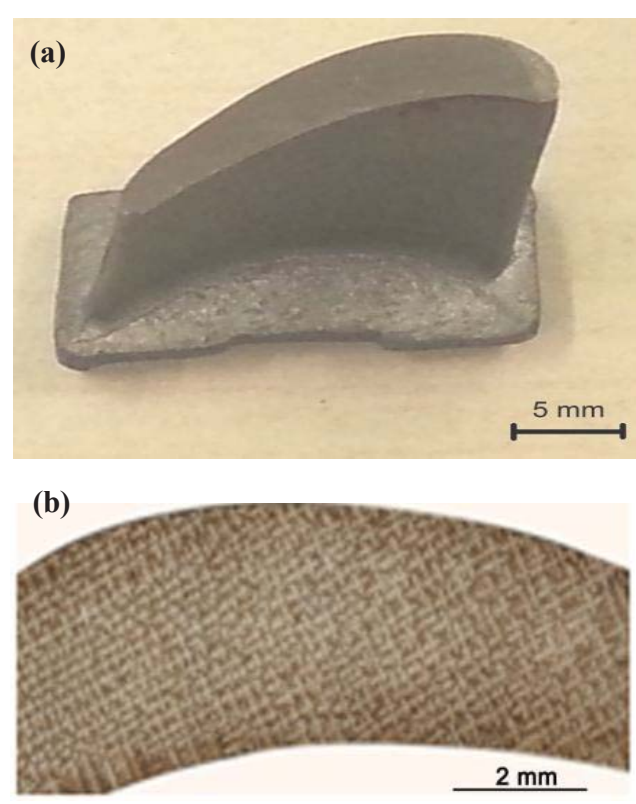

Figure 16. (a) a cast SC blade of superalloy CMSX-4 and (b) its microstructures.

Another important advantage of the D\&H technique is the complete avoidance of freckle formation. It is well-known that during the DS process of the superalloy components, the lighter elements such as $\mathrm{Al}$ and $\mathrm{Ti}$ segregate into the interdendritic regions, while the heavier elements such as $\mathrm{W}, \mathrm{Re}$, and $\mathrm{Co}$ partition to the dendrite core [19-25]. As the solidification proceeds, the alloy melt in the interdendritic regions at the bottom of the mushy zone becomes less heavy. In the conventional process the dendrites grow upward, leading to an inverse density distribution. This top-heavy melt in the mushy zone can result in thermosolutal convection which destroys the dendrites, thus 
leading to the formation of freckle defect. In the D\&H process, the interdendritic melt becomes top-light due to the downward solidification direction. This makes the mushy zone even more stable. As a result, the thermosolutal convection and the related freckle defects can be essentially eliminated by using the D\&H technique [26].

\section{Proposition for precise control of the SC growth in large blades}

As stated above, the solidification conditions in a complex blade are extremely inhomogeneous. This becomes more serious for large IGT parts. In the platform region, as shown in Figure 17 (a), the cooling at the outer extremities, $A_{1}$ and $A_{2}$, is more rapid than that in the inner corners, $\mathrm{B}_{1}$ and $\mathrm{B}_{2}$, where a heat barrier exists and hinders the SC growth from the blade body into the platform. As a result of the local undercooling, the stray grains could be formed at the extremities $A_{1}$ and $A_{2}$.

(a)

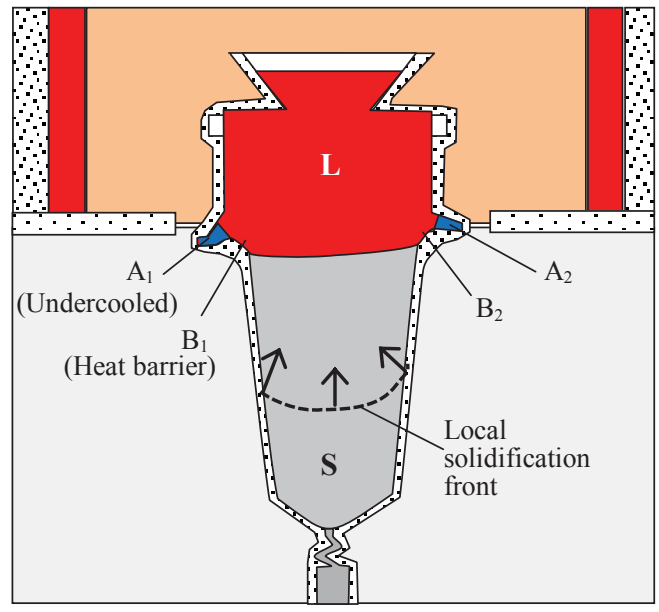

(b)

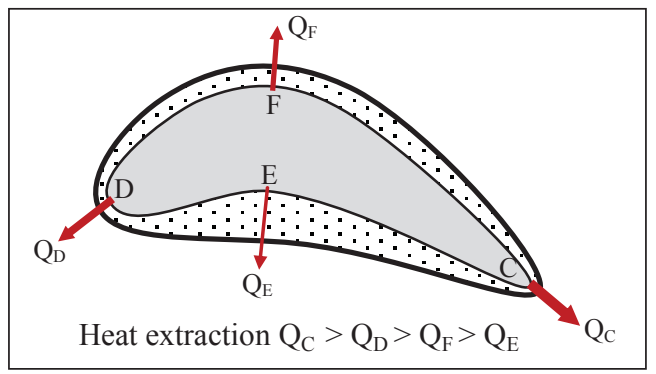

Figure 17. Schematics of (a) the longitudinal and (b) the cross section of a large turbine blade in a shell mold, exhibiting an inhomogeneous local cooling condition during the $\mathrm{SC}$ solidification.

Even in the blade body section where there is no abrupt change in the cross section, the cooling conditions of the blade surface are also inhomogeneous due to the different wall-thickness of the shell mold. As shown in Figure 17 (b), the trailing edge $\mathrm{C}$ experiences the best cooling condition because of the smallest wall-thickness of the shell mold, followed by the leading edge D. On the other hand, the concave surface E experiences the poorest local cooling conditions due to the thickest casting section, the largest wall-thickness of the mold as well as the worst view factor. In comparison, the heat extraction from the convex surface $\mathrm{F}$ is relatively easier. During the SC growth, the solidification front will pass through the blade body section with a concave curvature (Figure 17 (a)). Thus the local solidification direction will not be identically vertical, but will exhibit different inclinations, as indicated by the arrows in Figure 17 (a). In this case, some kinds of casting defects, such as freckles, stray grains and low angle grain boundaries (LABs), will be induced.

By applying the LMC or GCC techniques, the entire casting is more intensively cooled by a liquid metal bath or a cooling gas around its perimeter. The casting portions with smaller wallthickness of the shell mold, such as the extremities, $A_{1}$ and $A_{2}$, and the trailing edge $C$ (Figure 17), can be increasingly cooled, while in those portions having a larger wall-thickness, such as $B_{1}$ and $\mathrm{B}_{2}$ and $\mathrm{E}$, it can be relatively less affected. As a result, the inhomogeneous cooling in a casting becomes more pronounced and more unfavourable for a stable and controllable SC growth.

According to the above analysis, the SC growth in large blades is much more complex. Special efforts should be made to manage a proper lateral temperature distribution in order to precisely guide and control the SC solidification in three dimensions, rather than to generally improve the cooling conditions of an entire component.

\section{$\underline{\text { Targeted Cooling and Heating }(\mathrm{TCH})}$}

In order to achieve a proper thermal management for precise control of the SC growth in the critical regions of the IGT blades, a novel technology was proposed for Targeted Cooling and Heating (TCH). As shown in Figure 18 (a), the inner corners, $B_{1}$ and $\mathrm{B}_{2}$, are to be additionally cooled when the solidification front arrives at these positions in order to both remove the heat barrier in this region as well as to promote the lateral SC growth from the blade body into the platform. This can be achieved, for instance, by targeted spraying Ga-In alloy which remains liquid at room temperature. At the same time, the extremities, $A_{1}$ and $A_{2}$, should be additionally heated using lasers to overheat the local alloy melt until the SC growth from the blade body reaches into these extremities. As a result, a convex-shaped solidification front can be formed which is favorable for the lateral transition of the SC into the platform region, as shown in Figure 18 (a).

Furthermore, dynamic control of the SC growth can be achieved by simultaneously moving the targeted cooling and heating along a specified route from the blade body into the extremities of the platform. Based on the experimental data and numerical simulation, the intensity of cooling and heating will be controlled as a function of time and place in order to guide the three dimensional SC growth along an optimal route and sequence. Due to the establishment of a positive thermal gradient ahead of the local solidification front, the danger of the alloy melt's undercooling and the related grain defects can be completely removed. Even if the extremities have an overhanging geometry, such as at the extremity $A_{1}$ in Figure 18 (a), the local SC growth can still be precisely guided slightly downwards to the low-lying edges.

As shown in Figure 18 (b), the TCH method can also be applied to the blade body region of the IGT blades. Due to the poor cooling conditions, the concave $\mathrm{E}$ and convex F surfaces will be additionally cooled with more and less intensity, respectively. On the other hand, the trailing and leading edges which cool too rapidly should be additionally heated using lasers. The purpose 
here is to significantly reduce the concavity of the local solidification front (Figure 18 (a)), and to ensure a stable and homogeneous SC growth having the highest quality.

(a)

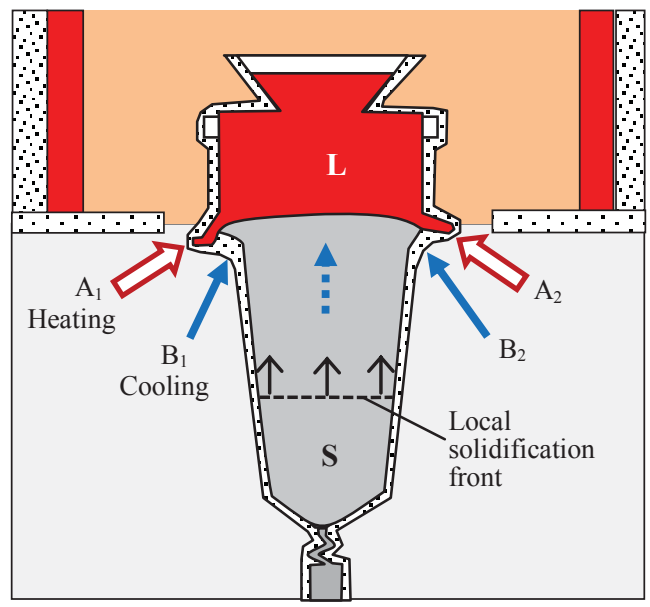

(b)

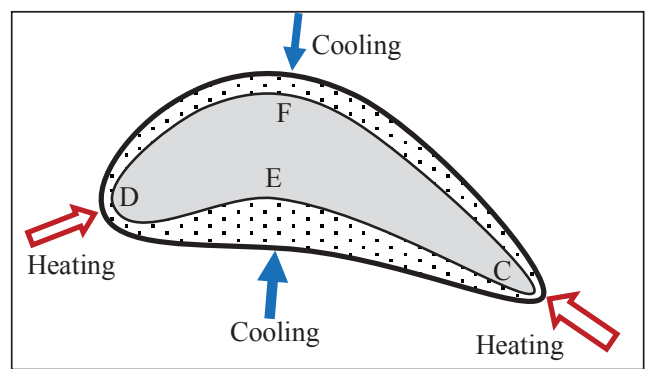

Figure 18. Schematics of the Targeted Cooling and Heating (TCH) technology to precisely control the SC solidification in large blades.

\section{Combination of TCH and D\&H Technologies}

As stated above, the D\&H Technology generally provides very good thermal conditions for the SC solidification due to the effective heat transfer. This technology can be further improved by combining it with the TCH technology, in which a proper lateral thermal arrangement can be established. In the D\&H process, as shown in Figure 19, the gas cooling is not applied from all directions, but is only aimed at the heat barrier zones where the lateral SC growth is hindered. At the platform extremities where the alloy melt cools rapidly, laser heating will be additionally used to prevent undercooling in these regions and, therefore, the formation of the stray grains. By optimally employing the $\mathrm{TCH}$ technique, the $\mathrm{SC}$ growth can be precisely guided along judicious routes to every edge of the blade. Due to the smaller wall-thickness of the shell mold in the D\&H process, the effect of the additional cooling and heating is more efficient and precise than that shown in Figure 18. As a result, besides the significant improvement in macroscopic cooling condition, a microscopic and dynamic control of the local SC growth in three dimensions can be achieved. Here, instead of injecting inert gas to strengthen the effectiveness of the process, the targeted cooling can be realized by dripping liquid Ar or He.

In the D\&H process, the TCH method can also be employed in the blade body region of the IGT blades. The concave $\mathrm{E}$ and convex $\mathrm{F}$ surfaces will be additionally cooled at different rates, while the trailing and leading edges should be correspondingly heated using lasers. Due to the significant reduction in the local solidification front's concavity, the SC growth in the blade body region can be improved to be more stable and homogeneous.

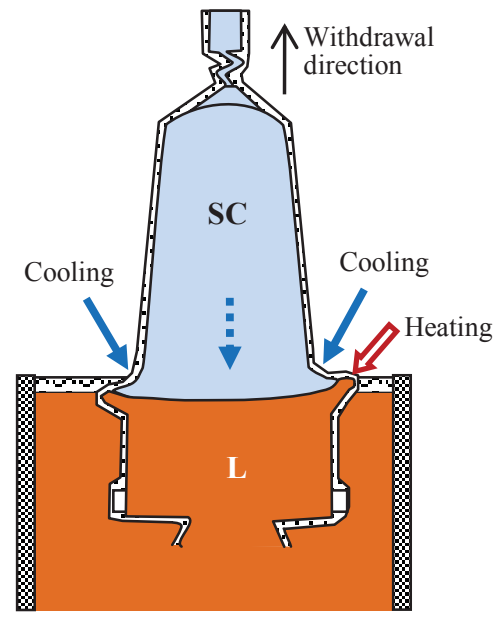

Figure 19. Schematic of the combination of the D\&H and TCH technologies.

\section{Summary}

A series of novel casting techniques were presented to show the contribution of the authors' institutions to the development in the production of the SC blades of superalloys. The Grain Continuator (GC) and the Heat Conductor (HC) technique were developed to circumvent or minimize the heat barrier in order to promote a successful transition of the SC growth from the blade body into the extremities of the platform. To achieve symmetric thermal conditions for solidifying the SC blades, the Parallel Heating and Cooling (PHC) system has been employed. Using this technique, both sides of a shell mold can be symmetrically heated in the heating zone as well as cooled in the cooling zone. The negative shadow effect in the current Bridgman process and the related defects are thereby significantly reduced. With the Dipping and Heaving (D\&H) technique using thin shell molds, the major problems with the Bridgman process, such as the ineffective radiative heat exchange and the large thermal resistance in the thick ceramic molds, can be effectively resolved. This technique also enables a freckle free solidification of the superalloy castings.

Based on the analysis of the solidification processing in complex turbine blades, a new concept of three dimensional and precise control of the SC growth was proposed. Correspondingly, the Targeted Cooling and Heating (TCH) technique was developed to establish a proper thermal arrangement for a microscopic control of the SC growth in the critical regions of the large IGT blades. By simultaneously moving the targeted cooling and heating along a specified route, a dynamic control of the SC growth can be achieved. Due to the establishment of a positive thermal gradient ahead of the local solidification front, the danger of the undercooling related grain defects can be completely removed. By employing the TCH together with the D\&H Technology, the additional cooling and heating becomes more sensitive and effective owing to the thinner shell molds. 


\section{References}

1. J.G. Tschinkel, A.F. Giamei, and B.H. Kearn, "Apparatus for casting of directionally solidified articles," U.S. Patent 3,763,926, 1973.

2. M. Konter, E. Kats, and N. Hofmann. "A novel casting process for single crystal gas turbine components," Superalloys 2000, ed. T.M. Pollock et al (Warrendale, PA: The Minerals, Metals and Materials Society, 2000), 189-200.

3. D. Ma, Q. Wu, and A. Bührig-Polaczek, "Undercoolability of superalloys and solidification defects in single crystal components," Advanced Materials Research, 278, (2011), 417422.

4. D. Ma, "Development of single crystal solidification technology for production of superalloy turbine blades," Acta Metallurgica Sinica, 51, (2015), 1179-1190.

5. M. Meyer ter Vehn, D. Dedecke, U. Paul, and P.R. Sahm. "Undercooling related casting defects in SC turbine blades," Superalloys 1996, ed. R.D. Kissenger et al (Warrendale, PA: The Minerals, Metals and Materials Society, 1996), 471-479.

6. D. Ma and A. Bührig-Polaczek, "Avoiding Grain Defects in Single Crystal Components by application of a Heat Conductor Technique" International Journal of Materials. Research, 100 (2009), 1145-1151.

7. D. Ma and A. Bührig-Polaczek, "Development of HeatConductor Technique for SC Components of Superalloys," International Journal of Cast Metals Research, 22, (2009), 422429 .

8. D. Ma and A. Bührig-Polaczek, "Application of HeatConductor Technique to Production of SC Turbine Blade," Metallurgical and Materials Transaction B, 40, (2009), 738-748.

9. J. Yu, Q.Y. Xu, K. Cui , B.C. Liu, A. Kimatsuka, Y. Kuroki, and A. Hirata, "Numerical Simulation of Solidification Process on Single Crystal Ni-Based Superalloy Investment Castings," Journal of Materials Science \& Technology, 23, (2007), 47-54.

10. R.E. Napolitano and R.J. Schaefer, "The convergence-fault mechanism for low-angle boundary formation in single-crystal castings," Journal of Materials Science, 35, (2000), 1641-1659.

11. D. Ma, Q. Wu, and A. Bührig-Polaczek, "Investigation on the asymmetry of thermal condition and grain defect formation in customary directional solidification process," IOP Conf. Series: Materials Science and Engineering, 27, (2011), 012037.

12. D. Ma, Q. Wu, and A. Bührig-Polaczek, "Some new observations on freckle formation in directionally solidified superalloy components," Metallurgical and Materials Transactions B, 43, (2012), 344-353.

13. D. Ma and A. Bührig-Polaczek, "The Influence of surface roughness on freckle formation in directionally solidified superalloy samples,"Metallurgical and Materials Transaction B, 43, (2012), 671-677.

14. D. Ma and A. Bührig-Polaczek, "The Geometry Effect of Freckle Formation in the Directionally Solidified Superalloy CMSX-4," Metallurgical and Materials Transactions A, 45, (2014), 1435-1444.
15. D. Ma, "A novel Bridgman-furnace construction for production of DS/SC blades of superalloys" (Report, ALD Vacuum Technologies GmbH, 2011).

16. D. Ma, H. Lu and A. Bührig-Polaczek, "Experimental Trials of the Thin Shell Casting (TSC) Technology for Directional Solidification," IOP Conf. Series: Materials Science and Engineering, 27, (2011), 012036.

17. F. Wang, D. Ma, J. Zhang, S. Bogner, and A. Bührig-Polaczek, "A high thermal gradient directional solidification method for growing superalloy single crystals," Journal of Materials Processing Technology, 214, (2014), 3112-3121.

18. F. Wang, D. Ma, S. Bogner, and A. Bührig-Polaczek, "Comparative investigation of the downward and upward directionally solidified single-crystal blades of superalloy CMSX4," Metallurgical and Materials Transactions A, DOI: 10.1007/s11661-016-3415-9.

19. D. Ma and U. Grafe, "Dendrite growth and microsegregation during directional solidification: an analytical model and experimental studies on the superalloys CMSX-4," International Journal of Cast Metals Research, 13, (2000), 85-92.

20. D. Ma and Uwe Grafe, "Microsegregation in directionally solidified dendritic-cellular structure of superalloy CMSX-4," Material Science and Engineering A, 270, (1999), 339-342.

21. Q. Feng, L.J. Carroll, and T.M. Pollock, "Solidification segregation in Ruthenium-containing nickel-base superalloys," Metallurgical and Materials Transactions A, 37, (2006), 19491962.

22. E.C. Caldwell, F.J. Fela, and G.E. Fuchs. "Segregation of elements in high refractory content single crystal nickel based superalloys," Superalloys 2004, ed. K.A. Green et al (Warrendale, PA: The Minerals, Metals and Materials Society, 2004), 811-818.

23. E.C. Caldwell, F.J. Fela, and G.E. Fuchs, "The segregation of elements in high-refractory-content single-crystal nickel-based superalloys," The Journal of The Minerals, Metals \& Materials Society, 56, (2004), 44-48.

24. F. Wang, D. Ma, S. Bogner, and A. Bührig-Polaczek, "Comparative study of the segregation behavior and crystallographic orientation in a nickel-based single-crystal superalloy," Journal of Alloys and Compounds, 647, (2015), 528532.

25. A. Heckl, R. Rettig, and R.F. Singer, "Solidification Characteristics and Segregation Behavior of Nickel-Base Superalloys in Dependence on Different Rhenium and Ruthenium Contents," Metallurgical and Materials Transactions A, 41, (2010), 202-211.

26. F. Wang, D. Ma, J. Zhang, and A. Bührig-Polaczek, "Investigation of segregation and density profiles in the mushy zone of CMSX-4 superalloys solidified during downward and upward directional solidification processes," Journal of Alloys and Compounds, 620, (2015), 24-30. 